\title{
The Role of Transverse Occipital Sulcus in Scene Perception and Its Relationship to Object Individuation in Inferior Intraparietal Sulcus
}

\section{Citation}

Bettencourt, Katherine C., and Yaoda Xu. 2013. The Role of Transverse Occipital Sulcus in Scene Perception and Its Relationship to Object Individuation in Inferior Intraparietal Sulcus. Journal of Cognitive Neuroscience 25, no. 10: 1711-1722.

\section{Published Version}

doi:10.1162/jocn_a_00422

\section{Permanent link}

http://nrs.harvard.edu/urn-3:HUL.InstRepos:12336274

\section{Terms of Use}

This article was downloaded from Harvard University's DASH repository, and is made available under the terms and conditions applicable to Other Posted Material, as set forth at http:// nrs.harvard.edu/urn-3:HUL.InstRepos:dash.current.terms-of-use\#LAA

\section{Share Your Story}

The Harvard community has made this article openly available.

Please share how this access benefits you. Submit a story.

\section{Accessibility}




\title{
The Role of Transverse Occipital Sulcus in Scene Perception and Its Relationship to Object Individuation in Inferior Intraparietal Sulcus
}

\author{
Katherine C. Bettencourt and Yaoda Xu
}

\begin{abstract}
The parietal cortex has been functionally divided into various subregions; however, very little is known about how these areas relate to each other. Two such regions are the transverse occipital sulcus (TOS) scene area and inferior intraparietal sulcus (IPS). TOS exhibits similar activation patterns to the scene selective parahippocampal place area, suggesting its role in scene perception. Inferior IPS, in contrast, has been shown to participate in object individuation and selection via location. Interestingly, both regions have been localized to the same general area of the brain. If these two were actually the same brain region, it would have important implications regarding these regions' role in cognition. To explore this, we first localized TOS and inferior IPS in individual participants and examined
\end{abstract}

\section{INTRODUCTION}

The parietal cortex has been shown to be a hub for a variety of cognitive processes, including attention (Behrmann, Geng, \& Shomstein, 2004; Yantis \& Serences, 2003; Corbetta \& Shulman, 2002; Culham \& Kanwisher, 2001; Kastner \& Ungerleider, 2000; Colby \& Goldberg, 1999), visual STM (Xu \& Chun, 2006, 2009; Todd \& Marois, 2004), numerical cognition (Hubbard, Piazza, Pinel, \& Dehaene, 2005; Dehaene, Piazza, Pinel, \& Cohen, 2003), motor planning (Buneo \& Andersen, 2006; Culham, Cavina-Pratesi, \& Singhal, 2006; Orban et al., 2006; Grefkes \& Fink, 2005; Merriam \& Colby, 2005; Gottlieb \& Goldberg, 1999), visual object individuation and identification (Xu \& Chun, 2006, 2009), and scene processing (Levy, Hasson, Harel, \& Malach, 2004; Grill-Spector, 2003; Hasson, Harel, Levy, \& Malach, 2003; Nakamura et al., 2000), with each of these cognitive operations being localized to specific parietal subregions. However, these regions have mostly been studied within isolated cognitive domains (e.g., activity in putative scene processing regions are only examined in tasks involving scenes), leaving it unclear whether the same parietal region is involved in different cognitive tasks. Research in other areas of the brain has given us numerous examples of singular regions that can be functionally localized using

Harvard University the degree of overlap between these regions in each participant. We found that TOS showed only a minor degree of overlap with inferior IPS $(\sim 10 \%)$. We then directly explored the role of TOS and inferior IPS in object individuation and scene perception by examining their responses to furnished rooms, empty rooms, isolated furniture, and multiple isolated objects. If TOS and inferior IPS were the same region, we would expect to see similar response patterns in both. Instead, the response of TOS was predominantly scene selective, whereas activity in inferior IPS was primarily driven by the number of objects present in the display, regardless of scene context. These results show that TOS and inferior IPS are nearby but distinct regions, with different functional roles in visual cognition.

a variety of tasks and stimuli, such as the STS (Grossman et al., 2000; Haxby, Hoffman, \& Gobbini, 2000; Bonda, Petrides, Ostry, \& Evans, 1996). Thus, our understanding of the functional organization of the human parietal cortex remains rather disjointed, leaving us wondering what relationships, if any, exist between parietal regions identified via different cognitive tasks. The inferior intraparietal sulcus (IPS) and the transverse occipital sulcus (TOS) scene area are prime examples of this problem.

Inferior IPS has been shown to play a role in the individuation of multiple objects by their locations (Xu, 2008, 2009; Xu \& Chun, 2006, 2009). TOS, on the other hand, appears to be involved in scene processing (Dilks, Julian, Paunov, \& Kanwisher, 2013; Levy et al., 2004; Grill-Spector, 2003; Hasson et al., 2003; Nakamura et al., 2000), showing similar patterns of activity to parahippocampal place area (PPA; Ward, MacEvoy, \& Epstein, 2010; Epstein \& Higgins, 2007; Epstein, Higgins, Jablonski, \& Feiler, 2007; Epstein, Higgins, \& Thomson-Schill, 2005; Levy et al., 2004; Hasson et al., 2003), and the retrosplenial complex (RSC; Epstein \& Higgins, 2007; Epstein et al., 2007; MacEvoy \& Epstein, 2007). Although PPA, RSC, and TOS have all been shown to be involved in scene processing, RSC does show some interesting differences from PPA and TOS. RSC shows stronger familiarity effects than TOS and PPA (Epstein et al., 2007), is insensitive to the retinotopic extent of objects (Troiani, Stigliani, Smith, \& Epstein, 2012), and lacks the eye

Journal of Cognitive Neuroscience 25:10, pp. 1711-1722 doi:10.1162/jocn_a_00422 
Table 1. Talairach Coordinates for Inferior IPS and TOS across a Variety of Studies

\begin{tabular}{|c|c|c|}
\hline \multirow[t]{4}{*}{ Inferior IPS } & Xu \& Chun, 2006 & $+26 /-21,-80 /-85,+30 /+26$ (off-center presentation) \\
\hline & & $+26 /-25,-65 /-70,+34 /+29$ (centered presentation) \\
\hline & $\mathrm{Xu}, 2008$ & $+27 /-21,-76 /-77,+28 /+25$ \\
\hline & Xu, 2009 & $+29 /-30,-78 /-82,+28 /+27$ \\
\hline \multirow[t]{9}{*}{ TOS } & Hasson et al., 2003 & $+33 /-34,-77 /-79,+12 /+12$ \\
\hline & Epstein \& Higgins, 2007 & $+32 /-33,-75 /-79,+34 /+31$ \\
\hline & Epstein et al., 2005 & $+40,-78,+22$ (right only) \\
\hline & Epstein et al., 2007 & $+36 /-42,-75 /-77,+24 /+26$ Familiarity effect (Exp. 1) \\
\hline & & $+31 /-36,-82 /-81,+20 /+19$ Viewpoint-specific adaptation (Exp. 1) \\
\hline & & $-45,-75,+23$ Familiarity effect, left only (Exp. 2) \\
\hline & & $+26 /-38,-80 /-80,+26 /+20$ Viewpoint-specific adaptation (Exp. 2) \\
\hline & & $-34,-85,+19$ Viewpoint-specific adaptation, new vs. old, left only (Exp. 2) \\
\hline & Levy et al., 2004 & $+32 /-34,-79 /-77,+14 /+16$ \\
\hline
\end{tabular}

centered coding that is seen in TOS and PPA (Ward et al., 2010). Thus, it has been suggested that TOS and PPA are primarily involved in the visual analysis of stimuli whereas RSC is more involved in either mnemonic processes related to scenes (Troiani et al., 2012) or to processing a scene relative to the broader environment (Ward et al., 2010). Little is known, however, about how processing in TOS differs from that of PPA. Only two studies, to our knowledge, have found significant differences between TOS and PPA. Troiani et al. (2012) found that, whereas PPA (and RSC) showed responses to object-based properties (like size, distance, etc.) when the objects were placed both within a scene context and on a white background, TOS showed object-based responses only when the objects were placed on a white background. The authors suggest that this may indicate that processing in TOS is related to the spatial qualities of individual objects. Dilks, Julian, Kubilius, Spelke, and Kanwisher (2011), on the other hand, found that, although TOS was sensitive to mirror reversals of scene stimuli, PPA was more tolerant, suggesting a split between scene recognition in PPA and navigation in TOS. However, both TOS and RSC showed similar sensitivity to mirror reversals. Thus, the true nature of processing within TOS remains unclear.

Despite the different functions ascribed to inferior IPS and TOS, both have been described as lying within the same general area of the brain, in the region where the IPS meets the TOS (see Table 1). The parietal cortex has also been shown to contain several topographic subregions, including V3A/B and IPS0 through IPS4 (Konen \& Kastner, 2008a, 2008b; Swisher, Halko, Merabet, McMains, \& Somers, 2007; Wandell, Dumoulin, \& Brewer, 2007; Schluppeck, Glimcher, \& Heeger, 2005; Silver, Ress, \& Heeger, 2005; see also Sereno, Pitzalis, \& Martinez, 2001). In particular, V3A/B has been localized to the same general area of both TOS and inferior IPS, namely at the base of IPS, where it transects the TOS (Orban, Van Essen, \& Vanduffel, 2004). Moreover, when directly compared with these topographic subregions, both inferior IPS and TOS appear to colocalize with V3B (Bettencourt \& Xu, in preparation; Nasr et al., 2011). All together, this suggests that inferior IPS and TOS may be the same brain region but are labeled differently in different tasks because of the way in which each region was localized.

If these brain regions were actually a singular region, it would substantially alter our understanding of the role of these regions in visual cognition. Given the individuation processes ascribed to inferior IPS and the recent finding of a preference for big, relative to small, objects within TOS (Konkle \& Oliva, 2012), it would suggest that the role of TOS in scene processing might be to individuate objects within a scene for further processing. This would be consistent with the findings from Troiani et al. (2012) and Dilks et al. (2011) and would provide perhaps a key distinction between PPA and TOS. However, this theory cannot be fully supported by the current state of the literature, as these two areas have been studied only in isolation, in separate groups of participants, with very specialized task paradigms. Given the strong individual differences seen in parietal structure (e.g., topographic regions in IPS; see Swisher et al., 2007), overlap between functional regions defined by group-averaged data could be easily inflated and/or obscured. As such, the precise relationship between TOS and inferior IPS can only be understood when comparisons are made within the same participants.

Thus, here, we localized both TOS and inferior IPS in the same individual participants with functional localizers previously established in the literature (see Xu \& Chun, 2006; Levy et al., 2004). We then determined the amount 
of overlap between TOS and inferior IPS. To further understand how these regions relate anatomically, we also examined the overlap between TOS and topographic IPS regions. Finally, to document the functional similarities and differences between these regions, we investigated the role of TOS and inferior IPS in scene perception and object individuation. Although scenes often contain multiple objects, they can also be impoverished and contain relatively few objects (i.e., an empty room). Previous research has shown that PPA is relatively insensitive to the total number of objects present within a scene (Epstein \& Kanwisher, 1998). However, if TOS colocalizes with inferior IPS and is involved in individuating objects in a scene, then its response should be high whenever multiple objects are present, regardless of whether a scene context is also present. On the other hand, if TOS is primarily a scene-processing region and is functionally distinct from inferior IPS, then it would show a high response to any type of scene stimuli, even when they contain very few objects, and a lower response to nonscene stimuli even when they contain many objects. The response of inferior IPS, on the other hand, should primarily reflect the number of objects present, regardless of the presence or absence of a scene context. Thus, by comparing activation patterns within TOS and inferior IPS to different types of stimuli, we should be able to see whether a functional distinction exists between them.

We found that despite of the proximity seen in groupaveraged studies, TOS and inferior IPS are both anatomically and functionally distinct regions. Even at a very liberal threshold of $p<.05$, the two regions showed a very small degree of overlap $(\sim 10 \%)$. Functionally, TOS was primarily scene driven and showed a low response to nonscene stimuli even when they contained many objects, whereas inferior IPS was driven by the presence of multiple objects in the display, independent of the presence a scene context. Together, these results show that TOS and inferior IPS are nearby, but distinct regions, with different roles in visual cognition.

\section{METHODS}

\section{Participants}

Eight paid participants (five women) from the Harvard University community were recruited to participate in this experiment. All participants gave informed consent in accordance with the institutional review board of Harvard University. Participants were between 22 and 34 years old (mean age $=28.6$ years). All had normal or corrected-to-normal visual acuity and were right-handed.

\section{Visual Stimuli and Experimental Paradigm}

Stimuli were presented by a Macintosh MacBook Pro to a liquid crystal display projected onto a screen mounted at the rear end of the scanner bore. Topographic mapping stimuli were presented using VisionEgg software (Straw, 2008), whereas stimuli for the main experiment were presented using Matlab with Psychtoolbox extensions (Brainard, 1997).

For the main experiment, participants were shown blocks (16 sec each) of digitized black and white photographs with 20 pictures per block. Each photograph subtended $13.2 \times$ $13.2^{\circ}$ of visual angle. Each block contained images from one of eight object categories: faces, scenes (outdoor and indoor), single isolated everyday objects, multiple isolated objects, furnished rooms, empty rooms, isolated furniture, and noise images (see Figure 1). Interspersed among stimuli blocks were five fixation blocks in which only a fixation dot was present throughout the entire block.

Stimuli used in the furnished room, empty room, and isolated furniture conditions were the exact same ones used by Epstein and Kanwisher (1998). Specifically, the furnished room stimuli consisted of unfamiliar indoor scenes with furniture, plants, and room decorations; empty rooms were the same rooms as the furnished rooms but with all furniture, plants, and room decorations removed, and each isolated furniture image consisted of the furnishings from one of the furnished rooms cut out from the original background, rearranged, and placed on a blank white background.

The multiple isolated objects stimuli used here consisted of the same shapes used in Xu and Chun (2006), but with a slightly different placement of the shapes. Previously, it has been shown that scenes activate a more peripheral representation than faces (Levy et al., 2004; Levy, Hasson, Avidan, Hendler, \& Malach, 2001). To ensure that this peripheral bias would not cause distortions in the localization of TOS relative to inferior IPS because of differences in the perceived eccentricity

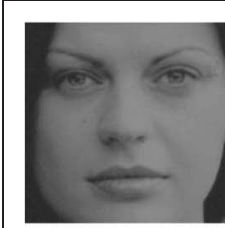

Faces

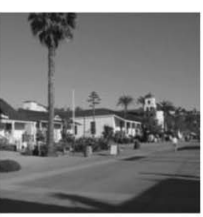

Scenes

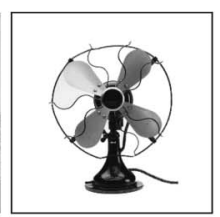

Single Isolated Objects

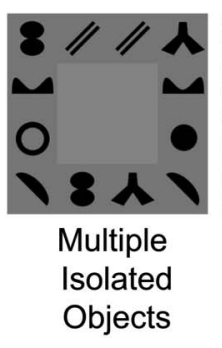

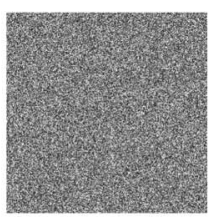

Noise

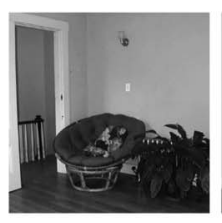

Furnished Rooms

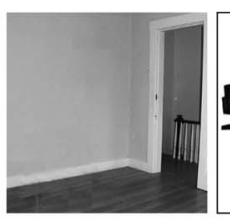

Empty

Rooms

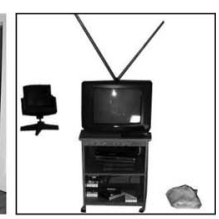

Isolated

Furniture

Figure 1. Example stimuli for each of the eight conditions used in the experiment. The furnished room, empty room, and furniture conditions were the exact same ones used in Epstein and Kanwisher (1998). 
of the stimulus, the multiple object stimuli here, unlike in $\mathrm{Xu}$ and Chun (2006), were presented at the peripheral most extent of the stimulus area and the number of stimuli was increased to 12 to ensure adequate coverage of this region (see Figure 1).

This collection of nine conditions (eight stimulus conditions plus one fixation condition) allowed us to localize TOS and inferior IPS, as well as examine each region's response to the presence of multiple objects both within and outside of a scene context. Trial and block design was based on Epstein and Kanwisher (1998). Each image was presented for $300 \mathrm{msec}$, followed by a 500-msec blank interval. To equate attention across conditions, participants were asked to detect a slight spatial jitter that would occur randomly throughout each block. A block of trials from each stimulus condition was presented twice during a run and block order was counterbalanced across runs (ABCD-EFGH-HGFE-DCBA for Version 1 and HGFE-DCBA-ABCD-EFGH for Version 2). Each participant completed two runs (each $5 \mathrm{~min}, 36 \mathrm{sec}$ ).

Topographic visual field representations of polar angle were also mapped for each participant, using between four and six runs (each $11 \mathrm{~min} 5.6 \mathrm{sec}$ ). Cortical representations of polar angle were mapped with flashing checkerboard stimuli using standard techniques (Swisher et al., 2007; Engel, Glover, \& Wandell, 1997; DeYoe et al., 1996; Sereno et al., 1995) with parameters optimized to reveal maps in the parietal cortex (Swisher et al., 2007). The polar angle wedge swept across the entire screen $(23.4 \times$ $17.5^{\circ}$ of visual angle), had an arc of $72^{\circ}$, flashed at $4 \mathrm{~Hz}$, had a sweep period of $55.467 \mathrm{sec}$, and swept out 12 cycles per run (for more details, see Swisher et al., 2007). The task varied slightly across participants. All participants were asked to detect a dimming in the visual display; for some participants the dimming occurred only at fixation, for others it occurred only within the polar angle wedge, and for some it could occur in both locations, commiserate with the various methodologies used in the literature (Bressler \& Silver, 2010; Swisher et al., 2007). No differences were seen in the maps obtained through each of these methods. We were able to identify in each participant areas within IPS including V3A, V3B, IPS0, IPS1, IPS2, IPS3, and IPS4. Other visual areas such as V1, V2, V3, V4, and MT could also be identified, but because our focus was on parietal areas, these other areas were not considered further in the present analysis.

\section{fMRI Methods}

The data were acquired on a Siemens Tim Trio 3T scanner with a 32-channel head coil at the Center for Brain Science at Harvard University (Cambridge, MA). Participants participated in two or three sessions of MRI scanning. In one session, a high-resolution $(1.0 \times 1.0 \times 1.3 \mathrm{~mm})$ anatomical image was collected for surface reconstruction. Before functional imaging in each session, T1-weighted echo-planar images were collected in the same slice pre- scription as the functional scans to allow each session to be registered to the participant's high-resolution anatomical scan. Functional data were acquired using T2*-weighted gradient-echo, echo-planar sequences. Each volume of the topographic data contained 42 slices (3 mm thick, $3.125 \times$ $3.125 \mathrm{~mm}$ in plane, no skip) oriented just off parallel from the AC-PC line to cover the full brain (repetition time = $2.6 \mathrm{sec}$, echo time $=30 \mathrm{msec}$, flip angle $=90^{\circ}$ ). Each volume of the main experimental data contained 24 slices ( $5 \mathrm{~mm}$ thick, $3.75 \times 3.75 \mathrm{~mm}$ in plane, no skip) parallel to the AC-PC line (repetition time $=2 \mathrm{sec}$, echo time $=$ 30 msec, flip angle $=90^{\circ}$ ).

\section{Data Analysis}

fMRI data were analyzed using the Freesurfer software package (Fischl, Liu, \& Dale, 2001; Dale, Fischl, \& Sereno, 1999; Fischl, Sereno, \& Dale, 1999). Data preprocessing included motion correction and intensity normalization. Computer representations of each cortical hemispheric surface were unfolded and inflated.

Parietal topographic maps were obtained by following the steps described in detail in Swisher et al. (2007). Scene selective PPA, RSC, and TOS ROIs were identified as areas that showed higher activity for scenes relative to both faces and single objects, as in Epstein and Kanwisher (1998). Inferior IPS was selected using the same procedure as Xu and Chun (2006) and consisted of the region that showed higher activation for multiple isolated objects relative to noise and that was located around the intersection of IPS and the TOS and around the Talairach coordinates previously reported (Xu, 2009; Xu \& Chun, 2006). All ROIs were defined independently of the data used for functional comparisons within and between ROIs in terms of object and scene processing. That is, contrasts used to define the ROIs were not analyzed further in the main analysis.

The significance thresholds for inferior IPS and TOS were initially set to a lenient $p<.05$ (uncorrected) to ensure that the totality of these regions would be selected and that any lack of overlap between them would not be because of an overly strict ROI definition. Additionally, a second set of inferior IPS and TOS ROIs were created using a stricter threshold ( $p<.001$, uncorrected) to examine whether the center of these two regions would overlap. The percentage of overlap between inferior IPS and TOS was calculated as the intersection of the two regions divided by the averaged size of these two regions (Kung, Peissig, \& Tarr, 2007). In other words, the area (in $\mathrm{mm}^{2}$ ) of overlap between inferior IPS and TOS divided by the average size of the whole TOS and whole inferior IPS regions, multiplied by 100 .

The amount of overlap between TOS and topographic regions was calculated as the percentage of TOS that overlapped with each topographic region. In other words, the area (in $\mathrm{mm}^{2}$ ) of overlap between TOS and each topographic region divided by the total area of TOS and then multiplied by 100. This percentage of 
overlap for each topographic region was then averaged across participants to get an average percentage of overlap between TOS and each topographic region. To obtain the total percentage of overlap between TOS and all of topographic regions, the average percentage per topographic region was summed together. The same procedure was used to calculate the overlap between inferior IPS and topographic regions. The overlap analyses between the topographic regions and inferior IPS and between the topographic regions and TOS were both done using the inferior IPS and TOS ROIs defined using both the lenient $p<.05$ and stricter $p<.001$ thresholds.

To compare the functional relationship between TOS and inferior IPS, fMRI response amplitudes in the furnished rooms, empty rooms, isolated furniture, and multiple isolated objects conditions were extracted within each ROI for each participant. By using data from these four conditions only, we were able to examine object and scene processing in our ROIs, while retaining independence from the data used for ROI definition. fMRI response amplitudes for each stimulus condition were measured in percent signal change, calculated by taking the difference in average signal intensity between each stimulus condition and the fixation condition, then dividing this difference by that of the fixation condition and multiplying it by 100. Left and right hemisphere ROIs were combined in our analysis as no response pattern difference was found between the two hemispheres. Data were analyzed in an individual subjects analysis approach. To account for response amplitude differences when comparing across ROIs, data were also normalized within each participant by dividing the response amplitude of each condition by that of the furnished room condition.

\section{RESULTS}

\section{Anatomical Overlap}

Overall, at the $p<.05$ (uncorrected) threshold, we found that there was a significant, but very small, overlap between the TOS and inferior IPS regions within individual participants (see Figure 2). On average, the two regions showed a $10 \%(S E=2.9)$ overlap, which was significantly greater than $0(t(7)=4.0, p<.01)$. We also examined the overlap between these two brain regions at the stricter threshold of $p<.001$ (uncorrected). At this threshold level, inferior IPS could not be localized in one participant in both hemispheres, and in the left hemisphere only for another participant. Excluding these two participants, in the remaining six participants, TOS and inferior IPS showed no overlap, indicating that the peak voxels for TOS and inferior IPS are in separate brain regions. There were no significant differences between the amount overlap between the left and right hemispheres $(p=.45)$. Overall, this indicates that, although Talairach coordinates from other studies have suggested that these two regions may colocalize, TOS and inferior IPS are actually anatomically distinct regions.

We then examined the overlap between both TOS and inferior IPS, separately, with the topographic regions at the $p<.05$ (uncorrected) threshold level. TOS showed, on average, a $49.8 \pm 10.0 \%$ of overlap with all parietal topographic areas, with $38.9 \pm 7.3 \%$ of TOS located within $\mathrm{V} 3 \mathrm{~B}, 2.6 \pm 1.8 \%$ in $\mathrm{V} 3 \mathrm{~A}$, and $8.2 \pm 4.4 \%$ in IPSO (see Figure $3 \mathrm{~B}$ ). The difference in percentage of overlap between the different parietal topographic regions and TOS reached significance, $F(2,14)=18.1, p<.001$, with more overlap seen between TOS and V3B than V3A and IPS0 ( $t \mathrm{~s}>4.5, p \mathrm{~s}<.01$ ) and no differences between the latter two $(p>.29)$. Replicating our previous work (Bettencourt \& Xu, in preparation), inferior IPS showed, on average, a $87.2 \pm 2.9 \%$ of overlap with all parietal topographic areas, with $40.0 \pm 7.6 \%$ of inferior IPS located within V3B, $28.8 \pm 6.9 \%$ in V3A, and $18.5 \pm 4.2 \%$ in IPSO (see Figure 3C). The difference in percentage of overlap between inferior IPS and the different parietal topographic regions did not reach significance $(p=$ .18). These findings suggest that TOS overlaps with the same topographic regions in IPS as suggested by Nasr et al. (2011) and the same topographic regions as inferior IPS. The latter confirms that TOS and inferior IPS are indeed located in the same general region of the brain. However, over half of TOS is located outside topographic regions, whereas $87 \%$ of inferior IPS is within these same topographic regions (this difference is statistically significant across our group of participants, $t(7)=3.78, p<$ $.01)$. This stark difference in the degree of overlap between these regions and topographic IPS again suggests that, although they are nominally in the same topographic area (V3A/B-IPSO region), TOS and inferior IPS cannot be the same brain region. Decreasing the significance threshold for TOS and inferior IPS ROIs to $p<.001$ (uncorrected) did not affect the degree of overlap between these regions and topographic IPS. Excluding the two participants whose inferior IPS could not be localized in both hemispheres at this threshold (see earlier description), inferior IPS showed a $89.4 \pm 3.5 \%$ overlap with all topographic regions, with $36.2 \pm 6.9 \%$ of inferior IPS in V3B, $39.2 \pm 9.0 \%$ in V3A, and $13.9 \pm 3.4 \%$ in IPSO. TOS could be localized in all participants at this threshold level and showed a $40.4 \pm 10.8 \%$ overlap with topographic regions, with $32.0 \pm 7.8 \%$ of TOS in V3B, $0.6 \pm$ $0.6 \%$ in V3A, and $7.9 \pm 6.8 \%$ in IPSO.

These findings demonstrate the importance of analyzing data within the same participants when comparing the precise locations of different brain regions. Using either Talairach coordinates or proximity to topographic regions, one would be led to assume that inferior IPS and TOS are highly colocalized brain regions; however, our individual subject approach, which better accounts for individual variations in the location of brain regions, clearly shows that these are two spatially separable regions. 
Figure 2. TOS (red), inferior IPS (blue), and their overlap (yellow) in all eight participants.

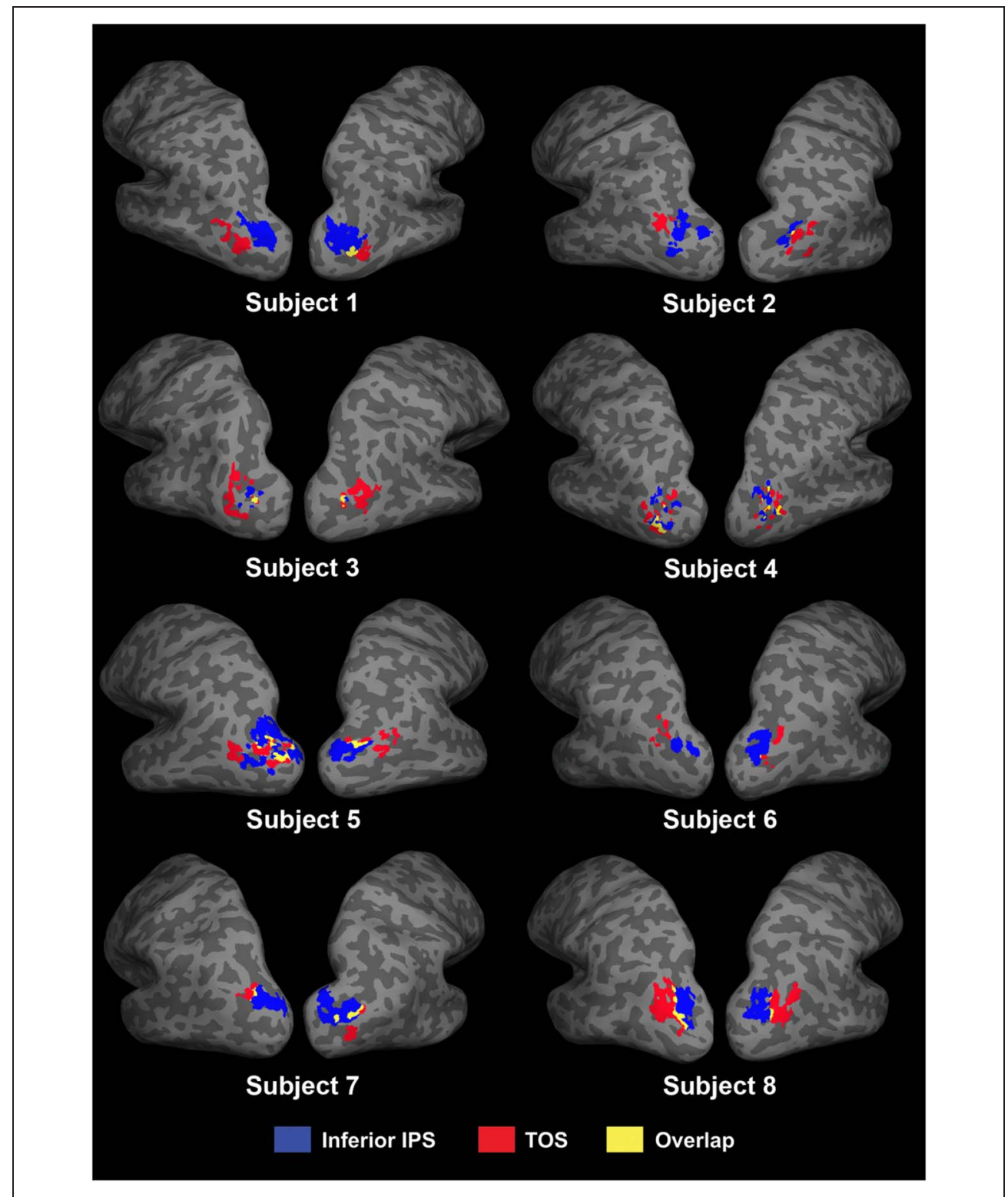

\section{Functional Differences}

Although our results suggest that TOS and inferior IPS are anatomically separable, this may be because of the way in which these areas are defined and may not actually represent a functional difference in the types of processing handled by these two regions. Thus, to determine whether there are functional differences between inferior IPS and TOS in how they process visual stimuli, we examined their responses to furnished rooms, empty rooms, isolated furniture, and multiple isolated objects. These four types of stimuli differ in the number of objects present, as well as the presence or absence of scene context, allowing us to examine the two processes that have been attributed to these regions in the literature, that of scene and object perception.

Behaviorally, performance on the motion detection task was high (84\% correct or higher) in each of these four stimulus conditions. There were no significant differences between conditions, although there was a slight trend toward higher performance in the multiple isolated objects condition (89.8\%) when compared with the empty rooms condition $(84.4 \% ; t(7)=2.11, p=.07)$.

As previous research has suggested that TOS plays a role in scene perception, we first compared activation in TOS with that of other scene areas, PPA and RSC (see Figure 4A and C). First, we examined the nonnormalized results within each ROI. All three scene areas showed significantly higher activation for scene stimuli (both furnished and empty) relative to both isolated furniture and multiple isolated objects (all $t \mathrm{~s}>2.9$, all $p \mathrm{~s}<.05$ ). All three also showed higher activation for isolated furniture relative to multiple isolated objects $(t \mathrm{~s}>4.0, p \mathrm{~s}<.01)$. PPA showed significantly higher activation for furnished rooms relative to empty rooms $(t(7)=2.5, p<.05)$, and TOS showed a similar trend $(t(7)=2.2, p=.07)$. 
There was no significant difference between empty and furnished rooms in RSC $(p>.3)$. Thus, for each of our scene areas, we observed higher activation for empty rooms compared with isolated furniture, showing a strong preference for scene stimuli within these regions. Our PPA results are consistent with those reported by Epstein and Kanwisher (1998), who also found a difference between the furnished and empty rooms and the isolated
Figure 3. (A) Topographic activation in a representative participant; (B) the location of TOS $(p<.05)$ and (C) inferior IPS $(p<.05)$ relative to topographic IPS regions in two representative participants.

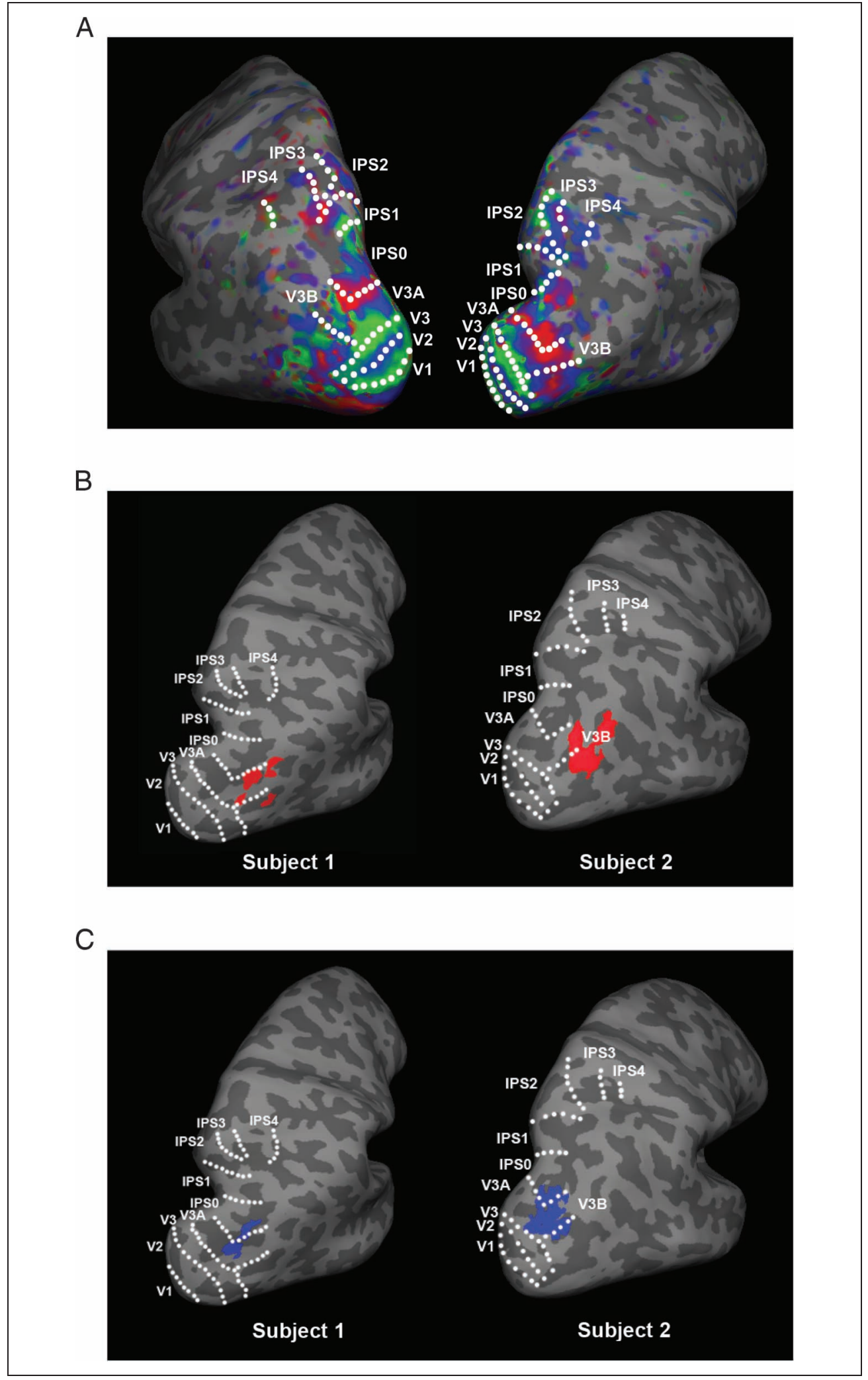


A

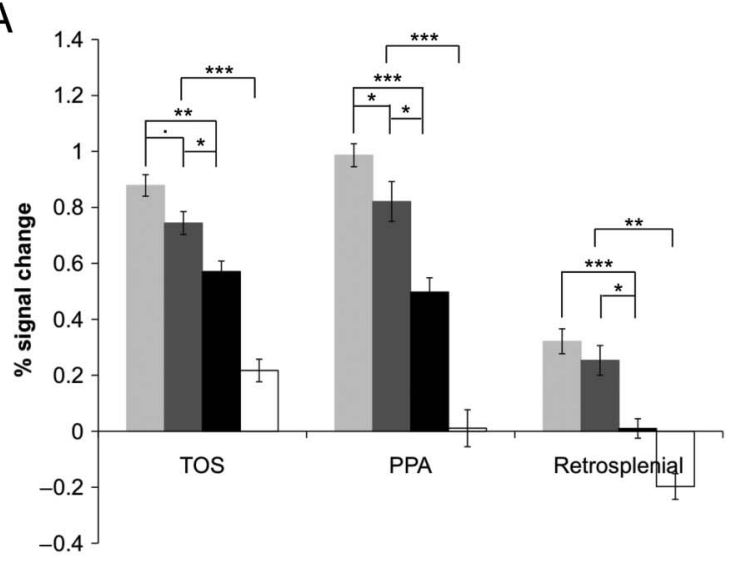

C

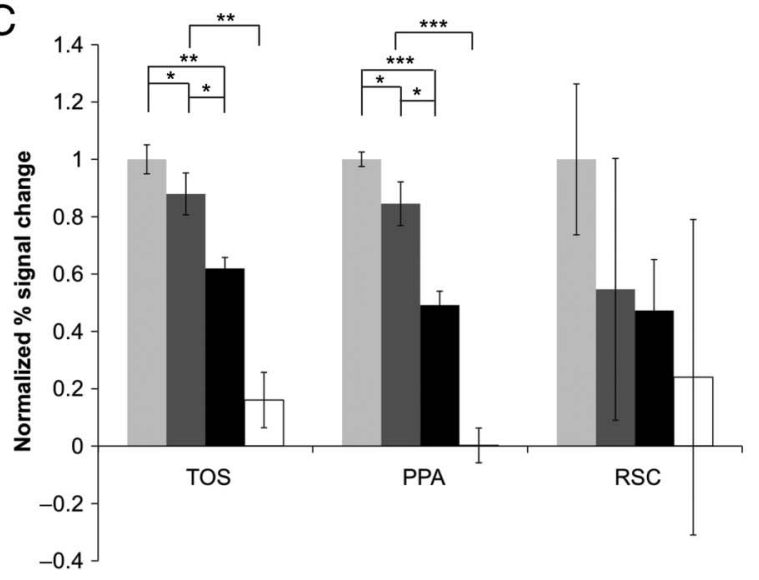

B

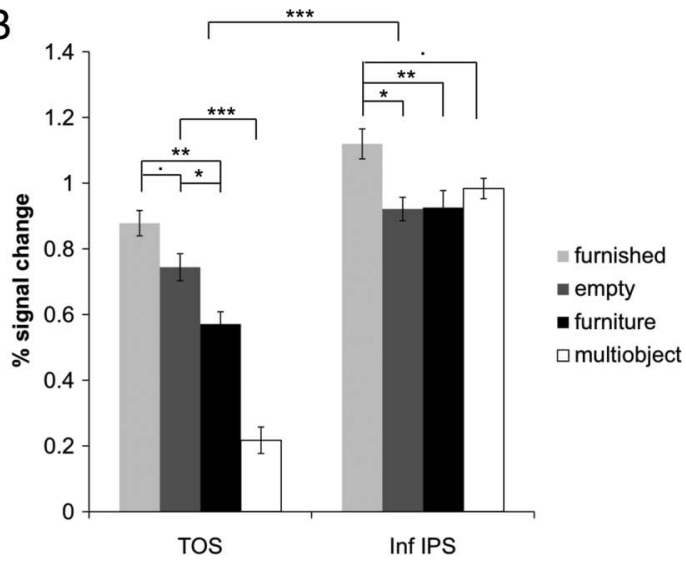

D

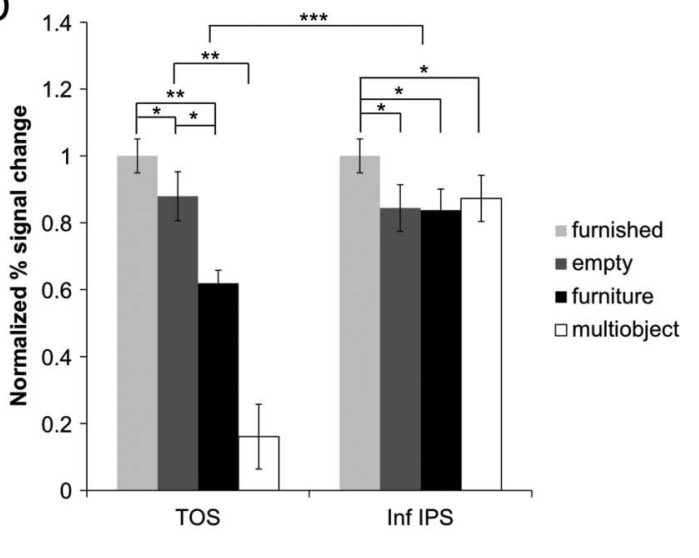

Figure 4. Nonnormalized functional activity for furnished rooms, empty rooms, isolated furniture, and multiple isolated objects in (A) the three scene areas and (B) TOS and inferior IPS. To account for response amplitude differences when comparing across ROIs and to facilitate between ROI comparisons, the data were also normalized within each participant by dividing the response amplitude for each condition by the furnished room condition. Normalized data are shown below for (C) the three scene areas and (D) TOS and inferior IPS. All three scene areas exhibited virtually the same response pattern, showing the lowest response to multiple isolated objects and preferring empty rooms to isolated furniture. Inferior IPS, on the other hand, showed similar responses to multiple isolated objects, empty rooms, and isolated furniture. A significant difference was found between TOS and inferior IPS in their responses to multiple isolated objects and in their response differences to empty rooms and isolated furniture.

furniture conditions; although they did not find a significant difference between the furnished rooms and the empty rooms conditions, a trend indicating this difference was present in their results.

We then normalized our data to account for any magnitude differences across the ROIs and compared response patterns across the three scene areas. The data were normalized by dividing the response amplitude for each condition by the furnished room condition. We found a main effect of stimulus condition, $F(3,21)=5.9$, $p<.01$, but no effect of ROI or interaction between ROI and condition ( $p s>.89$ ). This suggests that the pattern of responses across TOS, PPA, and RSC were similar, a finding that was supported in the nonnormalized detailed pairwise comparisons.

We then examined the response within inferior IPS (see Figure $4 \mathrm{~B}$ and $\mathrm{D}$ ). As in the scene areas, inferior IPS activation was higher for furnished rooms than for both isolated furniture and empty rooms ( $t \mathrm{~s}>2.6, \mathrm{ps}<.05)$. However, unlike the scene areas, inferior IPS showed no difference for empty rooms and isolated furniture $(p>9)$. We also found a high response in inferior IPS for multiple objects (as expected), and whereas this response was trending toward significantly less than that of furnished rooms $(t(7)=2.1, p=.07)$, it was no different to that of empty rooms or isolated furniture $(p s>.2)$. The trend toward higher activation for furnished rooms compared with multiple isolated objects was likely because of the fact that the former was more interesting and engaging than the latter. Moreover, the frequent repetition of the same set of objects within each block in the multiple isolated objects condition may have also caused a lowering of response amplitude because of fMRI adaptation, which would not have occurred in the furnished room condition.

Most importantly for the purpose of this study, we compared activity between inferior IPS and TOS in the four stimulus conditions, using normalized data to account for response magnitude differences between ROIs. 
We found a main effect of ROI, a main effect of condition, and a significant interaction between the two (all $F$ s $>$ 17.2 , $p \mathrm{~s}<.01)$. The latter indicates that the response patterns for the four stimulus conditions differed in these two brain regions (see Figure 4D). Specifically, the response differences between isolated furniture and both empty and furnished room conditions, as well as the differences between the multiple isolated objects condition and all other conditions, were greater in TOS than inferior IPS ( $t \mathrm{~s}>3.3, p s<.05)$. These results were robust and were seen in each of the eight participants tested. This suggests that TOS and inferior IPS differ significantly in how they represent scenes and objects.

Lastly, we examined whether the pattern of activation differed between regions of TOS that overlapped with topographic IPS regions and regions that did not. For one participant, there was no overlap between TOS and any of the topographic regions in one hemisphere and so, he was excluded from these analyses. Again, we used normalized data to account for response magnitude differences between ROIs. Here we found a main effect of stimulus condition, $F(3,18)=11.7, p<.001$, but no main effect of ROI (overlap vs. no overlap) or interaction between the two $(p>.4)$, and the overall result pattern mirrored that of the TOS results reported in Figure 4. Thus, whether or not TOS overlaps with topographic regions in IPS does not seem to affect the nature of its visual representation.

\section{DISCUSSION}

Previous research has placed both inferior IPS (Xu \& Chun, 2006, 2009) and TOS (Levy et al., 2004; Grill-Spector, 2003; Hasson et al., 2003; Nakamura et al., 2000) in the same general region of the brain, suggesting that these two regions may be one singular region. However, the work presented here clearly shows that, although these two regions are located within proximity to each other, they are separate regions, both anatomically and functionally. In spite of our very liberal statistical threshold for defining each ROI $(p<.05$, uncorrected), we found only a small percentage of overlap between the anatomical locations of these regions ( 10\%). Moreover, although inferior IPS was shown to be highly colocalized with topographic cortex (around $87 \%$ ), we found that over half of TOS is located outside topographic regions. This suggests that these are distinct brain regions that have only appeared to be colocalized across previous studies because of blurring caused by group-averaging and Talairach transformations.

Most importantly, TOS and inferior IPS showed functional differences in the processing of furnished rooms, empty rooms, isolated furniture, and multiple isolated objects. This set of conditions were chosen because they involved different amount of scene- and object-related processing, the two operations that have been associated with TOS and inferior IPS, respectively, in the literature. If TOS and inferior IPS were the same functional region, we would expect to see very similar response patterns in both. Instead, we saw distinct patterns of responses for processing scenes and multiple isolated objects in these two brain regions. Specifically, TOS showed a high response to any type of scene stimuli, even when they contain very few objects, and a much lower response to nonscene stimuli even when they contain many objects. This response pattern replicated a previous finding by Epstein and Kanwisher (1998) in PPA (another scene selective region) and is considered a hallmark of sceneselective processing in the brain. Inferior IPS, on the other hand, had a very different response pattern, showing a high level of activation whenever multiple objects were present, regardless of whether or not a scene context was also present. This indicates that TOS and inferior IPS are functionally distinctive in how they represent scenes and objects.

Although it may seem obvious that different functional contrasts would activate anatomically and functionally distinct regions, there are many counter examples. For instance, STS has been shown to be activated by both faces and biological motion (Grossman et al., 2000; Haxby et al., 2000; Bonda et al., 1996) and lower visual areas such as V1 can be activated by a variety of visual stimuli. Similarly, PPA is usually defined by contrasting scene and nonscene objects; however, this region also highly overlaps with regions defined using ensemble stimuli (Cant \& Xu, 2012). It is by understanding the plurality of stimuli and tasks that can activate a region that we understand the role of that region in cognition. Thus, this experiment provides a valuable first step toward understanding the relationship between TOS and inferior IPS.

Previous research has shown that inferior IPS participates in visual object individuation. This would predict that inferior IPS activation for isolated furniture should be higher than that for empty rooms, as by definition, the former would contain more objects than the latter. However, we found that inferior IPS activation did not differ between these two conditions. In our stimuli (which we took from Epstein \& Kanwisher, 1998), empty rooms were not simply blank walls and floors, but many also contained doorways, windows, outlets, etc. (see examples shown in Figure 5). Because the individuation and identification of these objects is likely important in scene recognition and navigation, they may be individuated in a manner similar to more standard objects (such as furniture or plants) in inferior IPS. This could explain the similarity in activity levels for empty rooms and multiple isolated objects, as well as the higher response to furnished rooms, which contained furniture as well as doorways, etc.

Although inferior IPS was localized by contrasting its response to multiple isolated objects to that of noise images, this brain region did not show the highest response to multiple isolated objects (the stimulus condition that defined it). But rather, it showed the highest response to furnished rooms. This was likely because of two reasons. First, the furnished room condition was 
Figure 5. Examples of the furnished room, empty room, and isolated furniture conditions showing the variety of stimuli used in each condition.

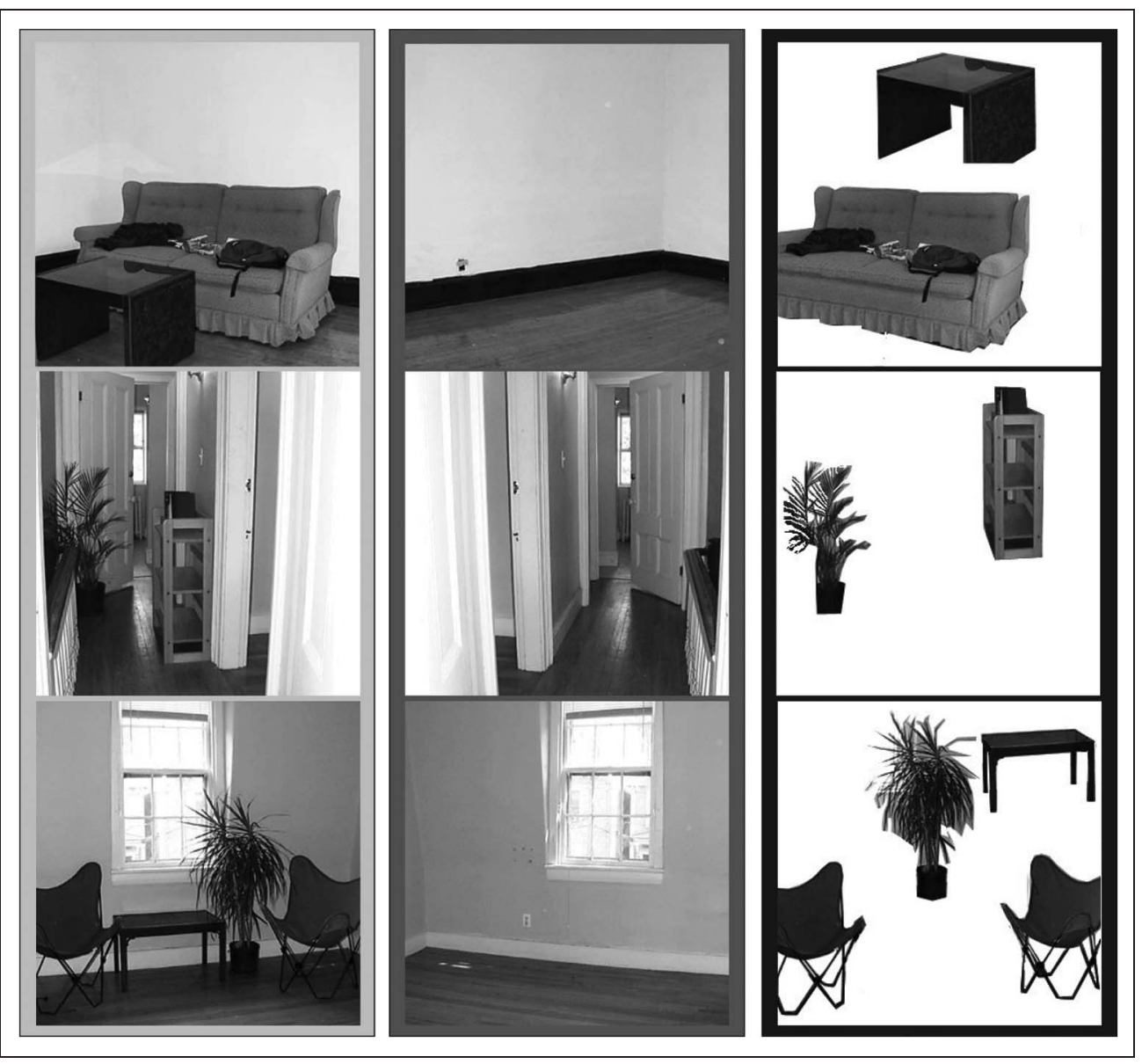

the most interesting, enriched, and engaging stimulus condition, which could have caused an increase in attention, and thus an increase in BOLD response. Second, in the multiple isolated objects condition, the same set of shapes were used repeatedly, albeit in different placements, in each trial. This likely resulted in fMRI adaptation effects and decreased the response for this condition compared with the other conditions. Note that these two effects were largely independent of the processing specificity of a brain region and would modulate responses in TOS as well in a similar manner. Despite the influence of these two effects, the pattern of response across all conditions varies significantly between inferior IPS and TOS, providing strong evidence that these are functionally distinctive brain regions.

One could argue that inferior IPS may only individuate objects in isolation, whereas TOS may individuate objects in a scene context, as this account would predict a pattern of TOS response similar to what we observed here (i.e., the highest response for the most number of objects present in a scene context (furnished rooms), the lowest response to isolated objects with no scene context (multiple isolated objects), and a moderately low response to object that have an implied scene context (isolated furniture)). However, it is unlikely that inferior IPS evolved to only individuate isolated objects, which are rarely encountered in the real world, and not also objects in a scene, which are seen everyday. Thus, it seems unnecessary for our brain to dedicate a separate brain region to individuate objects in a scene. Comparing the response of TOS in scene processing to other scene areas, we found that, although PPA, RSC, and TOS differed in their overall response amplitudes, they exhibited very similar response patterns (see Figure $4 \mathrm{~A}$ and $\mathrm{C}$ ), suggesting that all three regions participate in a similar manner in this aspect of scene perception. As such, it is insufficient to argue that TOS is involved in individuating objects in a scene but not PPA or RSC. Although the exact role of TOS in scene representation is unknown, the proximity of TOS to inferior IPS and its minor overlap with parietal topographic areas as found in this study, together with its position invariance related to the hemifield in which the stimuli are presented (MacEvoy \& Epstein, 2007) suggests that TOS is likely involved in higher-order spatial processing related to scenes, such as encoding the spatial relationships between objects within a scene.

More work is needed to explore the role of inferior IPS in individuation and to pinpoint the precise and unique role of TOS in scene processing relative to those of the other scene areas. Nevertheless, by more clearly localizing TOS in relationship to both inferior IPS and parietal topographic regions, the present findings represent an 
important step toward understanding the role of these brain regions in visual cognition.

\section{Acknowledgments}

We would like to thank Jonathan S. Cant and Sonia Poltoratski for their help in data collection, Jascha Swisher for his retinotopy code, and Nancy Kanwisher and Russell Epstein for sharing the scene stimuli from their 1998 publication. This research was supported by National Science Foundation Grant 0855112 to Y. X. and National Institutes of Health Grant F32-EY022874 to K. C. B.

Reprint requests should be sent to Katherine C. Bettencourt, Department of Psychology, Harvard University, 33 Kirkland Street, Room 720, William James Hall, Cambridge, MA 02138, or via e-mail: kcb@wjh.harvard.edu.

\section{REFERENCES}

Behrmann, M., Geng, J. J., \& Shomstein, S. (2004). Parietal cortex and attention. Current Opinion in Neurobiology, 14, 212-217.

Bettencourt, K. C., \& Xu, Y. (in preparation). The role of parietal topographic maps in visual object individuation and identification. Journal of Neuroscience.

Bonda, E., Petrides, M., Ostry, D., \& Evans, A. (1996). Specific involvement of human parietal systems and the amygdala in the perception of biological motion. Journal of Neuroscience, 16, 3737-3744.

Brainard, D. H. (1997). The psychophysics toolbox. Spatial Vision, 10, 433-436.

Bressler, D. W., \& Silver, M. A. (2010). Spatial attention improves reliability of fMRI retinotopic mapping signals in occipital and parietal cortex. Neuroimage, 53, 526-533.

Buneo, C. A., \& Andersen, R. A. (2006). The posterior parietal cortex: Sensorimotor interface for the planning and online control of visually guided movements. Neuropsychologia, 44, 2594-2606.

Cant, J. S., \& Xu, Y. (2012). Object ensemble processing in human anterior-medial ventral visual cortex. Journal of Neuroscience, 32, 7685-7700.

Colby, C. L., \& Goldberg, M. E. (1999). Space and attention in parietal cortex. Annual Review of Neuroscience, 22, 319-349.

Corbetta, M., \& Shulman, G. L. (2002). Control of goal-directed and stimulus-driven attention in the brain. Nature Reviews Neuroscience, 3, 201-215.

Culham, J. C., Cavina-Pratesi, C., \& Singhal, A. (2006). The role of parietal cortex in visuomotor control: What have we learned from neuroimaging? Neuropsychologia, 44, 2668-2684.

Culham, J. C., \& Kanwisher, N. G. (2001). Neuroimaging of cognitive functions in human parietal cortex. Current Opinion in Neurobiology, 11, 157-163.

Dale, A. M., Fischl, B., \& Sereno, M. I. (1999). Cortical surfacebased analysis. I. Segmentation and surface reconstruction. Neuroimage, 9, 179-194.

Dehaene, S., Piazza, M., Pinel, P., \& Cohen, L. (2003). Three parietal circuits for number processing. Cognitive Neuropsychology, 20, 487-506.

DeYoe, E. A., Carman, G. J., Bandettini, P., Glickman, S., Wieser, J., Cox, R., et al. (1996). Mapping striate and extrastriate visual areas in human cerebral cortex. Proceedings of the National Academy of Sciences, U.S.A., 93, 2382-2386.

Dilks, D. D., Julian, J. B., Kubilius, J., Spelke, E. S., \& Kanwisher, N. (2011). Mirror-image sensitivity and invariance in object and scene processing pathways. The Journal of Neuroscience, 31, 11305-11312.

Dilks, D. D., Julian, J. B., Paunov, A. M., \& Kanwisher, N. (2013). The occipital place area is causally and selectively involved in scene perception. The Journal of Neuroscience, 33, 1331-1336.

Engel, S. A., Glover, G. H., \& Wandell, B. A. (1997). Retinotopic organization in human visual cortex and the spatial precision of functional MRI. Cerebral Cortex, 7, 181-192.

Epstein, R. A., \& Higgins, S. (2007). Differential parahippocampal and retrosplenial involvement in three types of visual scene recognition. Cerebral Cortex, 17, 1680-1693.

Epstein, R. A., Higgins, S., Jablonski, K., \& Feiler, A. M. (2007). Visual scene processing in familiar and unfamiliar environments. Journal of Neurophysiology, 97, 3670-3683.

Epstein, R. A., Higgins, S., \& Thomson-Schill, S. L. (2005). Learning places from views: Variation in scene processing as a function of experience and navigational ability. Journal of Cognitive Neuroscience, 17, 73-83.

Epstein, R., \& Kanwisher, N. (1998). A cortical representation of the local visual environment. Nature, 392, 598-601.

Fischl, B., Liu, A., \& Dale, A. M. (2001). Automated manifold surgery: Constructing geometrically accurate and topologically correct models of the human cerebral cortex. IEEE Transactions on Medical Imaging, 20, 70-80.

Fischl, B., Sereno, M. I., \& Dale, A. M. (1999). Cortical surfacebased analysis. II: Inflation, flattening, and a surface-based coordinate system. Neuroimage, 9, 195-207.

Gottlieb, J., \& Goldberg, M. E. (1999). Activity of neurons in the lateral intraparietal area of the monkey during an antisaccade task. Nature Neuroscience, 2, 906-912.

Grefkes, C., \& Fink, G. R. (2005). The functional organization of the intraparietal sulcus in humans and monkeys. Journal of Anatomy, 207, 3-17.

Grill-Spector, K. (2003). The neural basis of object perception. Current Opinion in Neurobiology, 13, 159-166.

Grossman, E., Donnelly, M., Price, R., Pickens, D., Morgan, V., Neighbor, G., et al. (2000). Brain areas involved in perception of biological motion. Journal of Cognitive Neuroscience, $12,711-720$.

Hasson, U., Harel, M., Levy, I., \& Malach, R. (2003). Large-scale mirror symmetry organization of human occipito-temporal object areas. Neuron, 37, 1027-1041.

Haxby, J. V., Hoffman, E. A., \& Gobbini, M. I. (2000). The distributed human neural system for face perception. Trends in Cognitive Sciences, 4, 223-233.

Hubbard, E. M., Piazza, M., Pinel, P., \& Dehaene, S. (2005). Interactions between number and space in parietal cortex. Nature Reviews Neuroscience, 6, 435-448.

Kastner, S., \& Ungerleider, L. G. (2000). Mechanisms of visual attention in the human cortex. Annual Review of Neuroscience, 23, 315-341.

Konen, C. S., \& Kastner, S. (2008a). Representation of eye movements and stimulus motion in topographically organized areas of human posterior parietal cortex. Journal of Neuroscience, 28, 8361-8375.

Konen, C. S., \& Kastner, S. (2008b). Two hierarchically organized neural systems for object information in human visual cortex. Nature Neuroscience, 11, 224-231.

Konkle, T., \& Oliva, A. (2012). A real-world size organization of object responses in occipitotemporal cortex. Neuron, 74, 1114-1124.

Kung, C. C., Peissig, J. J., \& Tarr, M. J. (2007). Is region-of-interest overlap comparison a reliable measure of category specificity? Journal of Cognitive Neuroscience, 19, 2019-2034.

Levy, I., Hasson, U., Avidan, G., Hendler, T., \& Malach, R. (2001). Center-periphery organization of human object areas. Nature Neuroscience, 4, 533-539. 
Levy, I., Hasson, U., Harel, M., \& Malach, R. (2004). Functional analysis of the periphery effect in human building related areas. Human Brain Mapping, 22, 15-26.

MacEvoy, S. P., \& Epstein, R. A. (2007). Position selectivity in scene- and object-responsive occipitotemporal regions. Journal of Neurophysiology, 98, 2089-2098.

Merriam, E. P., \& Colby, C. L. (2005). Active vision in parietal and extrastriate cortex. Neuroscientist, 11, 484-493.

Nakamura, K., Kawashima, R., Sato, N., Nakamura, A., Sugiura, M., Kato, T., et al. (2000). Functional delineation of the human occipito-temporal areas related to face and scene processing - A PET study. Brain, 123, 1903-1912.

Nasr, S., Liu, N., Devaney, K. J., Yue, X., Rajimehr, R., Ungerleider, L. G., et al. (2011). Scene-selective cortical regions in human and nonhuman primates. Journal of Neuroscience, 31, 13771-13785.

Orban, G. A., Claeys, K., Nelissen, K., Smans, R., Sunaert, S., Todd, J. T., et al. (2006). Mapping the parietal cortex of human and non-human primates. Neuropsychologia, 44, $2647-2667$.

Orban, G. A., Van Essen, D., \& Vanduffel, W. (2004).

Comparative mapping of higher visual areas in monkeys and humans. Trends in Cognitive Sciences, 8, 315-324.

Schluppeck, D., Glimcher, P., \& Heeger, D. J. (2005). Topographic organization for delayed saccades in human posterior parietal cortex. Journal of Neurophysiology, 94, $1372-1384$.

Sereno, M. I., Dale, A. M., Reppas, J. B., Kwong, K. K., Belliveau, J. W., Brady, T. J., et al. (1995). Borders of multiple visual areas in humans revealed by functional magnetic resonance imaging. Science, 268, 889-893.

Sereno, M. I., Pitzalis, S., \& Martinez, A. (2001). Mapping of contralateral space in retinotopic coordinates by a parietal cortical area in humans. Science, 294, 1350-1354.
Silver, M. A., Ress, D., \& Heeger, D. J. (2005). Topographic maps of visual spatial attention in human parietal cortex. Journal of Neurophysiology, 94, 1358-1371.

Straw, A. D. (2008). Vision Egg: An open-source library for realtime visual stimulus generation. Frontiers in Neuroinformatics, 2, 4.

Swisher, J. D., Halko, M. A., Merabet, L. B., McMains, S. A., \& Somers, D. C. (2007). Visual topography of human intraparietal sulcus. Journal of Neuroscience, 27, 5326-5337.

Todd, J. J., \& Marois, R. (2004). Capacity limit of visual shortterm memory in human posterior parietal cortex. Nature, 428, 751-754.

Troiani, V., Stigliani, A., Smith, M. E., \& Epstein, R. A. (2012). Multiple object properties drive scene-selective regions. Cerebral Cortex, Epub ahead of print.

Wandell, B. A., Dumoulin, S. O., \& Brewer, A. A. (2007). Visual field maps in human cortex. Neuron, 56, 366-383.

Ward, E. J., MacEvoy, S. P., \& Epstein, R. A. (2010). Eye-centered encoding of visual space in scene-selective regions. Journal of Vision, 10, 6.

$\mathrm{Xu}, \mathrm{Y}$. (2008). Representing connected and disconnected shapes in human inferior intraparietal sulcus. Neuroimage, 40, 1849-1856.

Xu, Y. (2009). Distinctive neural mechanisms supporting visual object individuation and identification. Journal of Cognitive Neuroscience, 21, 511-518.

Xu, Y., \& Chun, M. M. (2006). Dissociable neural mechanisms supporting visual short-term memory for objects. Nature, 440, 91-95.

Xu, Y., \& Chun, M. M. (2009). Selecting and perceiving multiple visual objects. Trends in Cognitive Sciences, 13, 167-174.

Yantis, S., \& Serences, J. T. (2003). Cortical mechanisms of space-based and object-based attentional control. Current Opinion in Neurobiology, 13, 187-193. 